Home Search Collections Journals About Contact us My IOPscience

\title{
Development of Resistive Electrode Gas Electron Multiplier (RE-GEM)
}

This article has been downloaded from IOPscience. Please scroll down to see the full text article.

2012 JINST 7 C06006

(http://iopscience.iop.org/1748-0221/7/06/C06006)

View the table of contents for this issue, or go to the journal homepage for more

Download details:

IP Address: 128.183.97.246

The article was downloaded on 15/04/2013 at 18:51

Please note that terms and conditions apply. 
$2^{\text {nd }}$ International Conference on Micro Pattern Gaseous Detectors, 29 August - 1 SEPTEMBER 2011,

KOBE, JAPAN

\title{
Development of Resistive Electrode Gas Electron Multiplier (RE-GEM)
}

\author{
A. Yoshikawa, ${ }^{a, b, 1}$ T. Tamagawa, ${ }^{a, b}$ T. Iwahashi, ${ }^{a, b}$ F. Asami, ${ }^{a, b}$ Y. Takeuchi, ${ }^{a, b}$ \\ A. Hayato, ${ }^{c}$ H. Hamagaki, ${ }^{d}$ T. Gunji, ${ }^{a}$ R. Akimoto,${ }^{d}$ A. Nukariya, ${ }^{d}$ S. Hayashi, ${ }^{d}$ \\ K. Ueno, ${ }^{a}$ A. Ochi ${ }^{e}$ and R. Oliveira ${ }^{f}$ \\ ${ }^{a}$ RIKEN, \\ 2-1, Hirosawa, Wako, Saitama 351-0198, Japan \\ ${ }^{b}$ Department of Physics, Tokyo University of Science (TUS), \\ 1-3, Kagurazaka, Shinjuku-ku, Tokyo, 162-8601, Japan \\ ${ }^{c}$ NASA Goddard Space Flight Center (GSFC), \\ 8463, Greenbelt, Rd Greenbelt, MD 20770-2548, U.S.A. \\ ${ }^{d}$ Center for Nuclear Study (CNS), Graduate School of Science, Universe of Tokyo, \\ 7-3-1, Hongo, Bunkyo-ku, Tokyo, 113-0033, Japan \\ ${ }^{e}$ Department of Physics, Kobe University, \\ 1-1, Rokkodai, Nada, Kobe, Japan \\ ${ }^{f}$ CERN European Organisation for Nuclear Research, \\ Geneva 23, Switzerland \\ E-mail: akifumi@crab.riken.jp
}

Abstract: We successfully produced Resistive-Electrode Gas Electron Multiplier (RE-GEM) which has resistive electrodes instead of the metal ones which are employed for the standard GEM foils. RE-GEM has a resistive electrode of $25 \mu \mathrm{m}$-thick and an insulator layer of $100 \mu \mathrm{m}$-thick. The hole structure of RE-GEM is a single conical with the wider and narrower hole diameters of $80 \mu \mathrm{m}$ and $60 \mu \mathrm{m}$, respectively. A hole pitch of RE-GEM is $140 \mu \mathrm{m}$. We obtained the maximum gain of about 600 and the typical energy resolution of about 20\% (FWHM) at an applied voltage between the resistive electrodes of $620 \mathrm{~V}$, using a collimated $8 \mathrm{keV}$ X-rays from a generator in a gas mixture of $70 \% \mathrm{Ar}$ and $30 \% \mathrm{CO}_{2}$ by volume at the atmospheric pressure. We measured the effective gain as a function of the electric field of the drift region and obtained the maximum gain at an drift field of $0.5 \mathrm{kV} / \mathrm{cm}$.

KeYwords: Gaseous detectors; Micropattern gaseous detectors (MSGC, GEM, THGEM, RETHGEM, MHSP, MICROPIC, MICROMEGAS, InGrid, etc)

\footnotetext{
${ }^{1}$ Corresponding author.
} 


\section{Contents}

1 Introduction 1

2 Production of RE-GEM 2

3 Test setup 3

4 Experiments and results $\quad 5$

4.1 Energy resolution and gain of RE-GEM 5

4.2 Dependence of gain on $E_{\mathrm{d}}$

5 Summary 9

\section{Introduction}

Gas Electron Multiplier (GEM) foil fabricated by F. Sauli in 1996 is one of the micro-pattern gas detectors (MPGD) [1]. The widely-used GEM has a structure, like a parallel plate condenser. Dense through holes are drilled in an insulator substrate (thickness is about $50 \mu \mathrm{m}$ ) sandwiched by thin copper foils (thickness is about $5 \mu \mathrm{m}$ ). For example, so-called "CERN standard GEM" has a hole pitch of $140 \mu \mathrm{m}$ and a diameter of $70 \mu \mathrm{m}$. The electron multiplication occurs inside the holes when we apply the voltage $(500-600 \mathrm{~V})$ between the electrodes in an appropriate gas. An advantage of using GEM is that we can obtain high gas gain, which reaches $10^{6}$ in a stacked GEM configuration. On the other hand, the disadvantage is a fragility against sparks, in common with MPGD [2]. Figure 1 shows a magnified photograph of a burned out GEM. The aforementioned discharge may cause a short circuit between the GEM electrodes and break GEM. Thus, a new GEM has been developed, which can reduce the discharge energy by the electrical resistance. It is named Resistive GEM, since it has the resistive material for electrodes instead of copper [3, 4].

There are two types of Resistive GEM which have been studied so far: Resistive Electrode Thick GEM (RETGEM) and Resistive Mesh GEM (RM-GEM). RETGEM is originally developed by Mauro et al. [3, 5], replacing the copper electrodes of Thick GEM with resistive electrodes. The gas gain of single RETGEM reaches the same value as that of Thick GEM; the maximum gain is about $10^{5} \mathrm{~s}$. To keep the position resolution of GEM, we need Resistive GEM with a fine pitch. However, the pitch and the hole diameter of RETGEM are coarse: $700 \mu \mathrm{m}$ and $300 \mu \mathrm{m}$ respectively (figure 2a). The mechanical drilling is used to drill holes in RETGEM because of the thickness of RETGEM substrate $(500 \mu \mathrm{m})$. It is possible but challenging to make holes with a diameter of $100 \mu \mathrm{m}$ mechanically.

Oliveira et al. developed another kind of Resistive GEM, RM-GEM, which has much finer pitch and hole diameter than RETGEM [4]. Figure 2b shows the structure of RM-GEM. RMGEM is composed of two resistive electrode meshes which have the fine pitch; the hole diameter is 


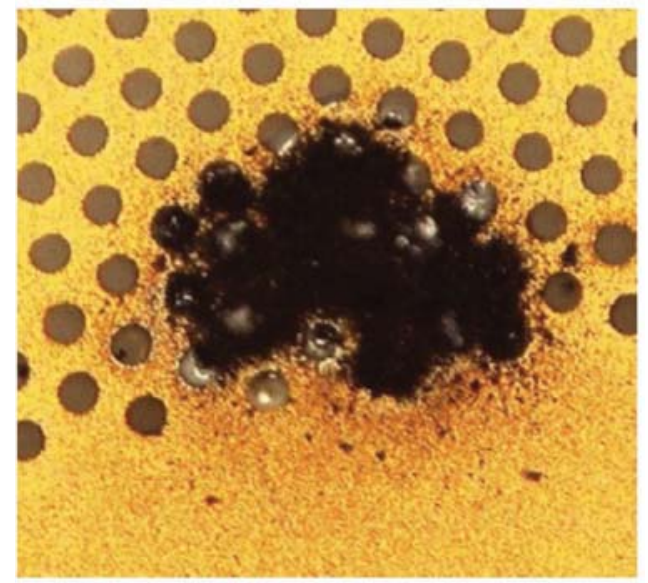

Figure 1. A magnified photograph of a burned out GEM. (a)

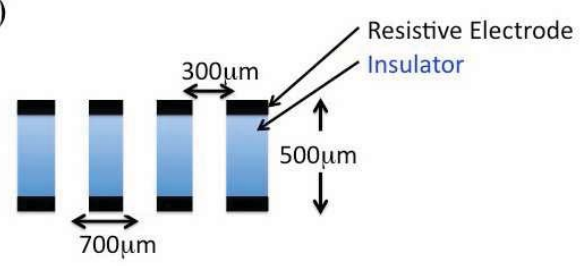

(b)

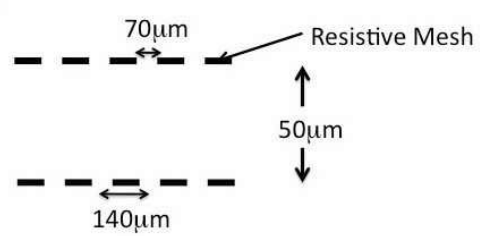

Figure 2. The structures of (a) RETGEM, and (b) RM-GEM.

$70 \mu \mathrm{m}$ and the pitch is $140 \mu \mathrm{m}$. The two resistive electrodes are separated in $50 \mu \mathrm{m}$ and there is no insulator layer between mesh electrodes. They succeeded to make RM-GEM with the fine pitch, however the gas gain is about 50 [4]. The gain is limited due to the discharge [4].

In order to produce a Resistive GEM with the fine pitch and the high gain, we have the advantage of that we already developed fine pitch and high gain GEM with $100 \mu \mathrm{m}$-thick LCP (LCPGEM) by laser etching techniques [6, 7]. Therefore, we started replacing the copper electrode of LCP-GEM with resistive material. It is called Resistive Electrode GEM (RE-GEM). Hereafter in this paper, we deal with LCP-GEM as a standard metal electrode GEM, because the performances of LCP-GEM is the same as those of CERN standard GEM [6, 7].

\section{Production of RE-GEM}

Figure 3 shows a schematic diagram of the RE-GEM production. First, we made the substrate of RE-GEM from Resistive-Kapton and bonding sheets by heating and pressuring (figure 3a). The surface resistivity of Resistive-Kapton is a few $M \Omega / \square$. The bonding sheet is polyimide with a lower melting point than that of Resistive-Kapton. Second, we drilled holes through the substrate by focused laser with a cone shape. The laser was irradiated from one side and the number of drilled holes by one shot was 60 (figure 3b). At last, we cleaned the smear developed by the laser etching process inside the holes (figure $3 \mathrm{c}$ ).

Figures 4 show the structures of RE-GEM. The thickness of RE-GEM, which is $150 \mu \mathrm{m}$, is about third times smaller than that of RETGEM. The structure of RE-GEM consists of three layers: $25 \mu \mathrm{m}$ Resistive-Kapton and $100 \mu \mathrm{m}$ bonding sheet. The distance between electrodes of RE-GEM is $100 \mu \mathrm{m}$. The hole structure of RE-GEM is a single conical, the larger hole diameter is $80 \mu \mathrm{m}$ and the smaller hole diameter is $60 \mu \mathrm{m}$. The pitch of holes is $140 \mu \mathrm{m}$. 


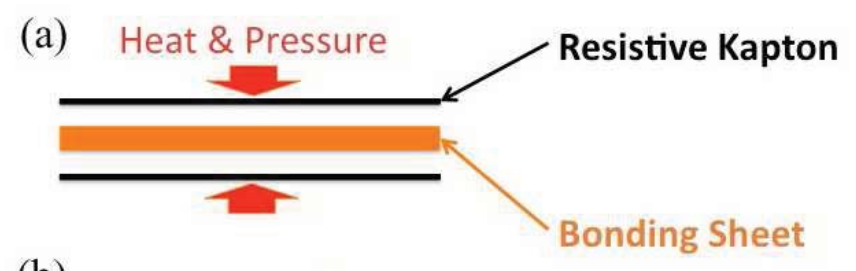

(b)

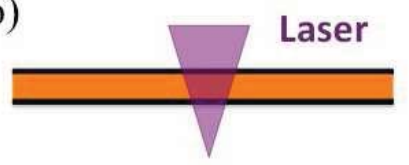

(c)

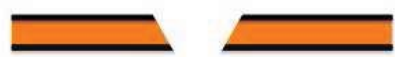

Figure 3. The schematic view of creative processes: (a) Creating substrate process. (b) Laser etching process. (c) Cleaning smear in holes process.

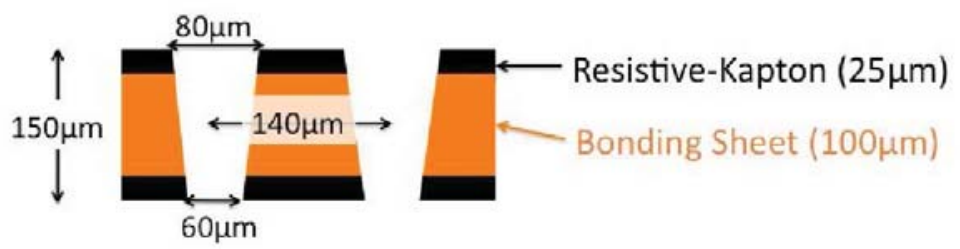

Figure 4. The structure of RE-GEM.

\section{Test setup}

Figure 5 shows a schematic view of the experimental setup. The setup contained a drift plane, an RE-GEM foil, and a readout pad. The drift plane, RE-GEM, and the readout pad have an equal effective area of $26 \times 26 \mathrm{~mm}^{2}$. Figure 6 shows a photograph of RE-GEM. The drift plane and RE-GEM was $5.5 \mathrm{~mm}$ apart, and this volume is the drift region, where the X-ray is absorbed. The electric field of the drift region $\left(E_{\mathrm{d}}\right)$ was set to $0.5 \mathrm{kV} / \mathrm{cm}$. The readout pad was placed $1 \mathrm{~mm}$ under the RE-GEM. The volume between the readout pad and the RE-GEM foil is called induction region. The electric field of this region $\left(E_{\mathrm{i}}\right)$ varied $6.0-8.0 \mathrm{kV} / \mathrm{cm}$. The drift plane, RE-GEM, and the readout pad were placed in the gas chamber which was filled with a gas mixture of $70 \% \mathrm{Ar}$ and $30 \% \mathrm{CO}_{2}$ by volume $\left(\mathrm{Ar} / \mathrm{CO}_{2}=70 \% / 30 \%\right)$. The gas mixture was flowing through the system at the atmospheric pressure. We used $5.9 \mathrm{keV} \mathrm{X}$-rays from an ${ }^{55} \mathrm{Fe}$ radioactive source which was placed outside the chamber or a pencil beam of $\mathrm{Cu} \mathrm{K} \alpha \mathrm{X}$-rays $(8 \mathrm{keV})$ from a generator. The incoming $\mathrm{X}$-ray got through a Kapton window of the chamber. The pencil beam of X-ray was collimated to a diameter of $200 \mu \mathrm{m}$. The count rates of the incident X-ray from the ${ }^{55} \mathrm{Fe}$ radioactive source and the generator were about 40 and 80 counts/sec, respectively. A high voltage was supplied via a chain 


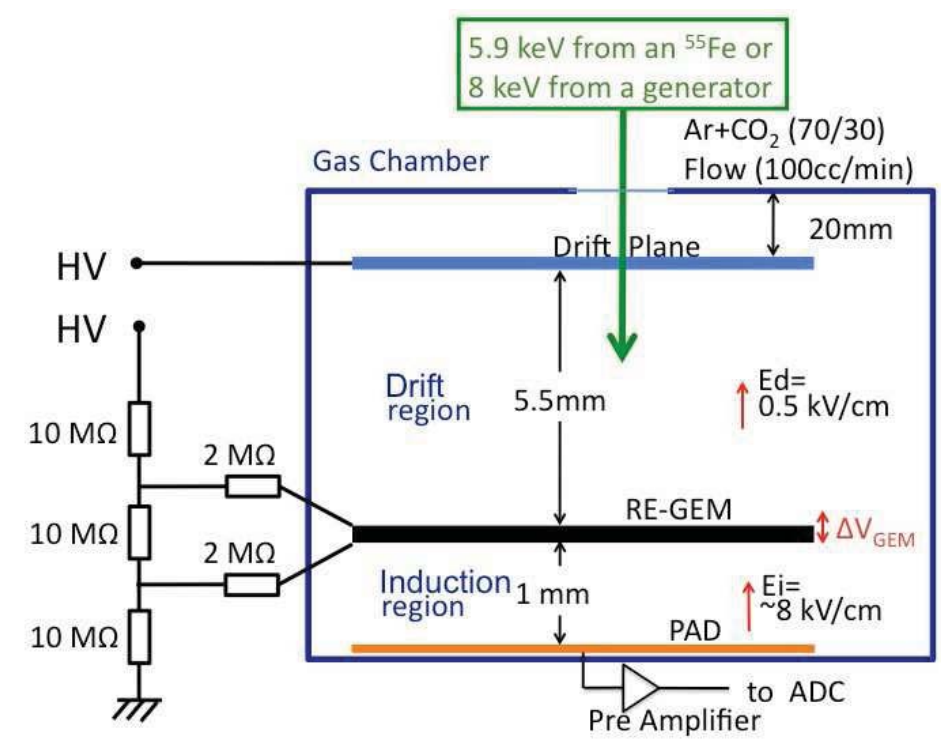

Figure 5. A schematic view of the test setup.

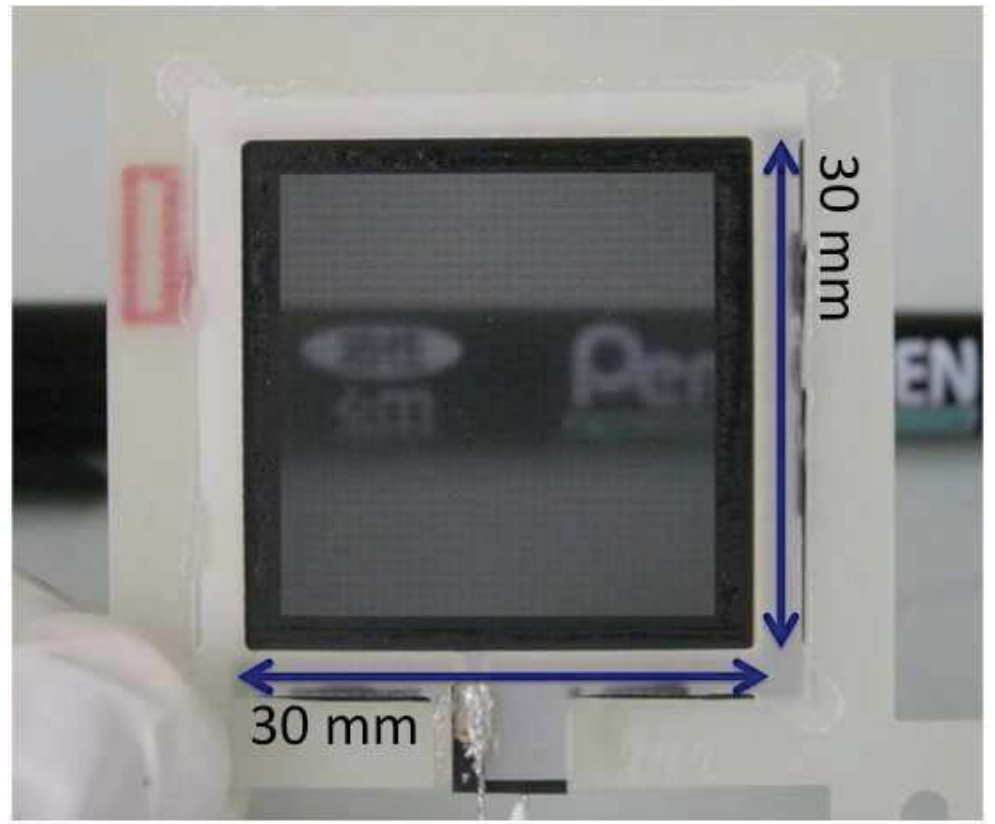

Figure 6. A photograph of RE-GEM. The effective area is $26 \times 26 \mathrm{~mm}^{2}$.

of $10 \mathrm{M} \Omega$ resistors. We also had the $2 \mathrm{M} \Omega$ resisters for protections.

The charge signals amplified by RE-GEM were fed into a preamplifier (AmpTek A225) which contained a shaper $1 \mu \mathrm{sec}$. The signal from the preamplifier was fed into a main custom amplifier 
which had both features of delay and discriminator. The signal amplified by the custom amplifier was converted to a digital data by a peak-hold analog-digital converter.

\section{Experiments and results}

\subsection{Energy resolution and gain of RE-GEM}

We measured the energy resolution of RE-GEM with $E_{\mathrm{d}}$ of $0.5 \mathrm{kV} / \mathrm{cm}$ and $E_{\mathrm{i}}$ of $6.0-8.0 \mathrm{kV} / \mathrm{cm}$. REGEM was mounted with the wide holes towards the drifting electrons (W-N setup) or the narrow holes towards the drifting electrons (N-W setup). Figures $7 \mathrm{a}$ and $7 \mathrm{~b}$ show the ADC spectra taken with the W-N and N-W setups at an applied voltage of $600 \mathrm{~V}$, respectively. The incident X-ray was provided by a generator with a $\mathrm{Cu}$ target $(8 \mathrm{keV})$ and collimated to a diameter of $200 \mu \mathrm{m}$. The peak corresponding to $8 \mathrm{keV}$ was clearly seen in both figures $7 \mathrm{a}$ and $7 \mathrm{~b}$. The energy resolution of RE-GEM taken with the W-N and N-W setups were $23 \%$ and $19 \%$ in FWHM.

The intrinsic gas gain inside the amplified channels was probably identical between the W-N and N-W setups when the applied voltage was identical. However the amplified electrons collected by the anode electrode with the W-N setup was larger than that with the N-W setup, because more electric field lines with the W-N setup encountered to the anode electrode than those with the N-W setup [8]. The energy resolution with the W-N setup became worse than that with the N-W setup due to more loss of signal electrons by the anode.

The energy resolution of RE-GEM with the W-N and N-W setups using the non-collimated $\mathrm{X}$-rays of $5.9 \mathrm{keV}$ from ${ }^{55} \mathrm{Fe}$ were $30 \%$ and $82 \%$, respectively. Those value are worse than those of CERN standard GEM and LCP-GEM $(\Delta E / E \sim 20 \%)[6,7]$. The worse energy resolution probably comes from the non-uniformity of the effective gas gain on RE-GEM; the homogeneity of the hole geometry of RE-GEM may not be perfect as LCP-GEM, because we drilled the holes by laser oneby-one. Although the energy resolution using the collimated X-rays with the W-N setup was worse than that with the N-W setup, the energy resolution using the non-collimated X-rays with the W-N setup was better than that with the N-W setup. The reason was probably that the uniformity of the effective gas gain with the W-N setup was better than that with the N-W setup. However we have not investigated the gain uniformity of RE-GEM yet.

We measured the effective gas gain as a function of an applied voltage between electrodes (gain curve) of RE-GEM, using the non-collimated X-rays of $5.9 \mathrm{keV}$ from ${ }^{55} \mathrm{Fe}$. The effective gas gain $(G)$ is calculated as follows;

$$
G=\frac{\mu}{q_{e} \cdot n_{e}},
$$

where $\mu$ is the peak value which we find as a peak in the ADC spectrum, $q_{e}$ is the elementary charge and $n_{e}$ is the number of electrons created by the absorption of a $5.9 \mathrm{keV}$ X-ray. For $\mathrm{Ar} / \mathrm{CO}_{2}=70 \% / 30 \%$ at the atmospheric pressure, $n_{e}$ is 212 [9]. We hence after call the effective gas gain just the gain.

Figure 8 shows the gain curve of RE-GEM taken with the W-N and N-W setups. For comparison, we plotted the gain curve of LCP-GEM, which has an equal thickness $(100 \mu \mathrm{m})$ of insulator to RE-GEM, since the gain of GEM depends on the electric field strength in holes and the distance between electrodes. The detailed structure of LCP-GEM is shown in figure 9. The gain of LCPGEM was obtained with $E_{\mathrm{d}}$ of $2.5 \mathrm{kV} / \mathrm{cm}$ and $E_{\mathrm{i}}$ of $5.5-7.5 \mathrm{kV} / \mathrm{cm}$ in $\mathrm{Ar} / \mathrm{CO}_{2}=70 \% / 30 \%$. The 


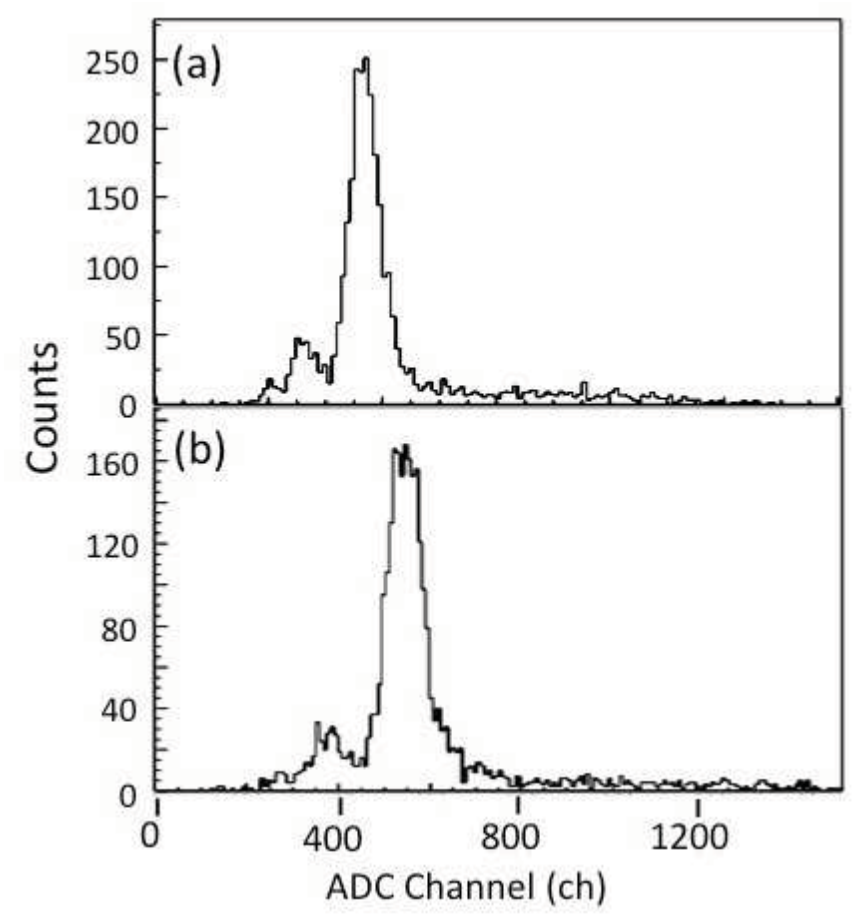

Figure 7. (a) The ADC spectrum of RE-GEM for the W-N setup and (b) the ADC spectrum of RE-GEM for the $\mathrm{N}-\mathrm{W}$ setup, at an applied voltage of $600 \mathrm{~V}$ using the pencil beam from a generator with a main emission line at $8 \mathrm{keV}$.

slope of the gain curves of RE-GEM and LCP-GEM were almost identical each other, indicating that the electric field inside the multiplication channel was also identical. The normalization of the gain curve of RE-GEM taken with the N-W setup was similar to that of LCP-GEM. The maximum gain of RE-GEM was about 600 . The normalization of the gain curve of RE-GEM taken with the $\mathrm{N}-\mathrm{W}$ setup is about 1.5 times as large as that taken with the W-N setup. This trend is commonly seen for GEM foils with the conical hole structure [10].

We have reported the experimental results of another RE-GEM and the noisy signals whose origin was unknown in a conference record (unknown signals) [11]. The count rate of the unknown signals reported in the record increased rapidly at an applied voltage of $720 \mathrm{~V}$. Although there were the unknown signals, the count rate of the unknown signals was very low in this experiment. We could not apply voltage over $660 \mathrm{~V}$ to this RE-GEM, thus we could not find the high rate signals like the unknown signals reported in the record. We note that the gain of RE-GEM in this report was higher at lower applied voltage than that of RE-GEM reported in the record [11].

\subsection{Dependence of gain on $E_{\mathrm{d}}$}

Figure 10 shows the RE-GEM gain as a function of $E_{\mathrm{d}}$ taken with the N-W and W-N setups at the applied voltage of $620 \mathrm{~V}$ and $E_{\mathrm{i}}$ of $6.2 \mathrm{kV} / \mathrm{cm}$. Figure 11 shows the electron transparency of REGEM, where the electron transparency has been normalized to unity, with the implicit assumption 


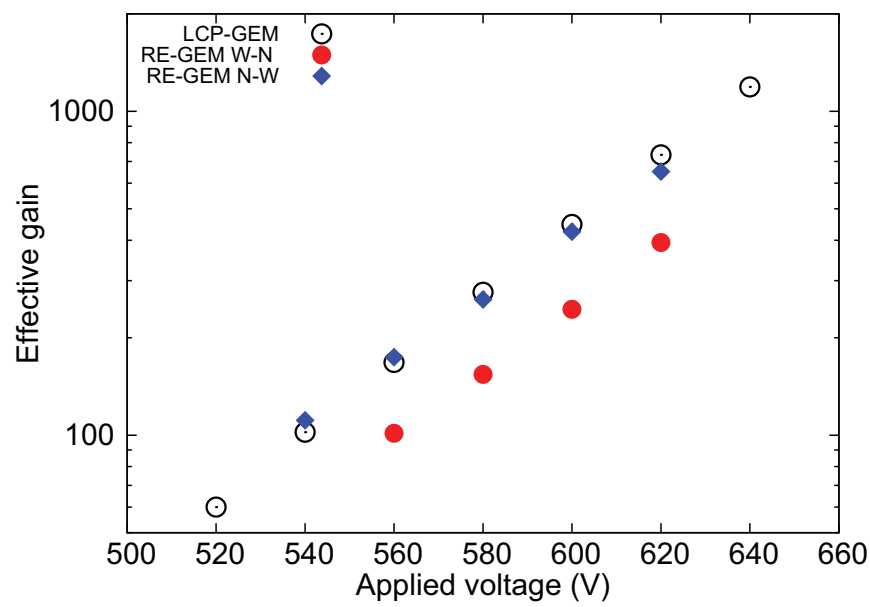

Figure 8. The gain curves of RE-GEM taken with the W-N and N-W setups, and LCP-GEM.

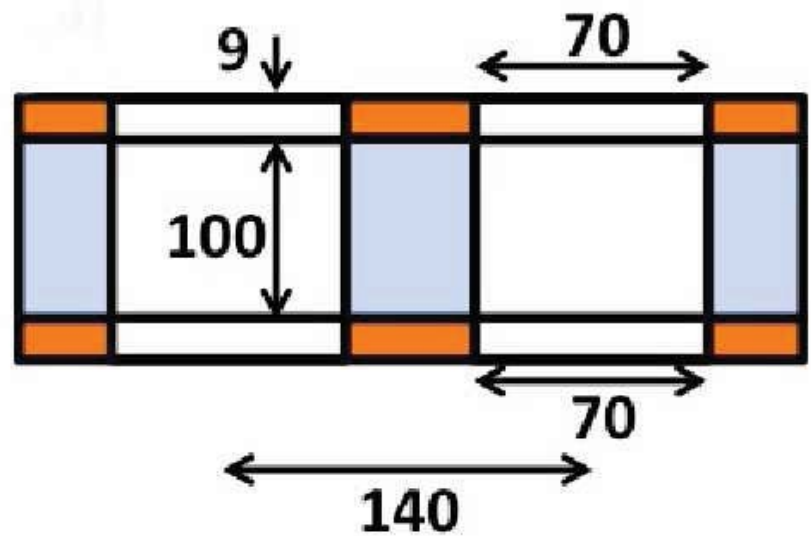

Figure 9. The structure of LCP-GEM.

of full transparency in the plateau region. We employed the definition of the electron transparency described in the reference [8]. For comparison, we superposed the electron transparency of LCPGEM in the figure 11. The data of LCP-GEM was obtained in $\mathrm{Ar} / \mathrm{CO}_{2}=70 \% / 30 \%$ with an applied voltage of $630 \mathrm{~V}$ and $E_{\mathrm{i}}$ of $6.3 \mathrm{kV} / \mathrm{cm}$. The electron transparency of RE-GEM taken with the W-N and N-W setups decreased from the unity above $E_{\mathrm{d}}=0.5 \mathrm{kV} / \mathrm{cm}$, while the electron transparency of LCP-GEM decreased from the unity above $E_{\mathrm{d}}=2.0 \mathrm{kV} / \mathrm{cm}$. RE-GEM is more opaque for drift electrons than LCP-GEM.

At the unity of the electron transparency, all lines of electric force in the drift region pass through the GEM holes by its definition. When $E_{\mathrm{d}}$ is larger than $2.0 \mathrm{kV} / \mathrm{cm}$ where the electron transparency of LCP-GEM decreases from unity, some part of the lines of electric force in the drift region encounter the LCP-GEM cathode. The electron transparency of RE-GEM decreased 


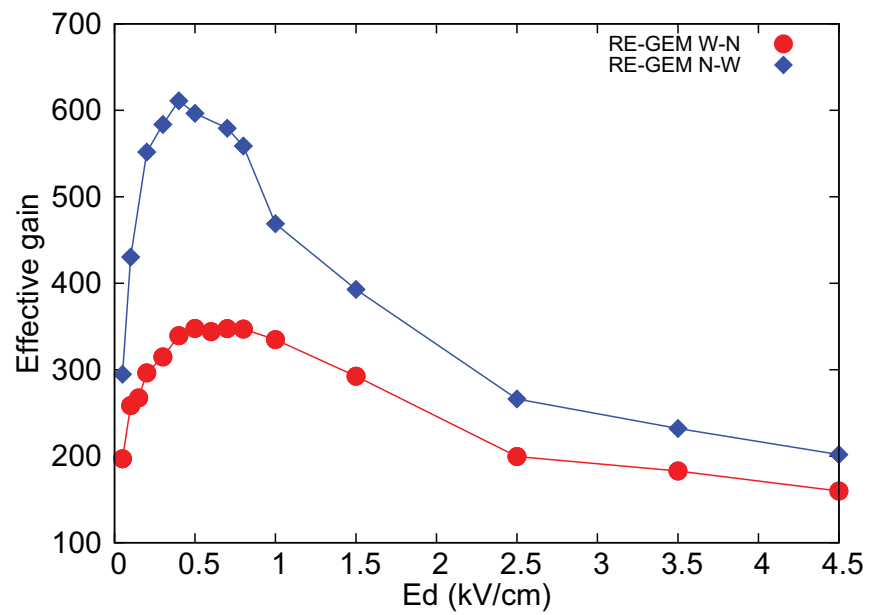

Figure 10. The effective gain of RE-GEM as a function of $E_{\mathrm{d}}$ with the W-N and N-W setups.

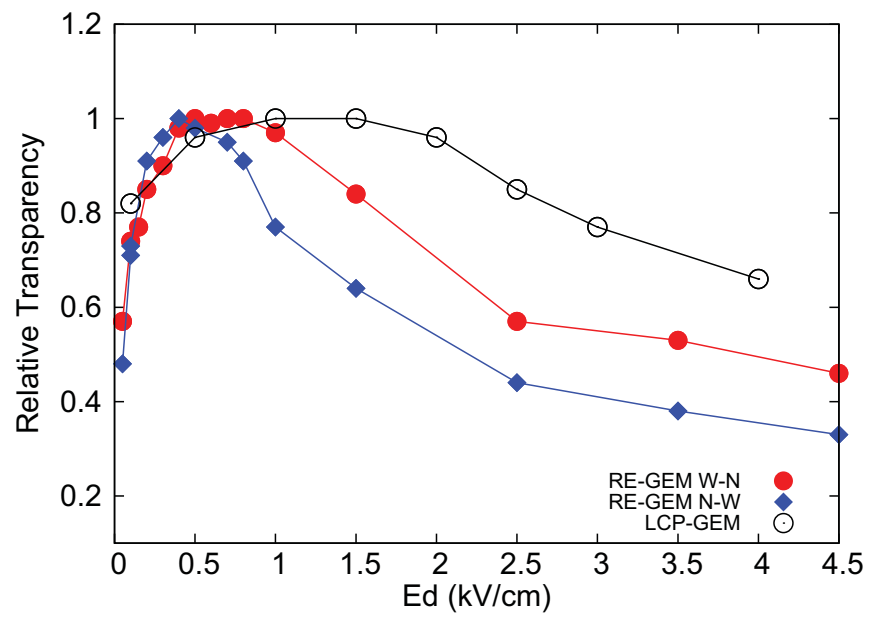

Figure 11. The electron transparency of RE-GEM as a function of $E_{\mathrm{d}}$ with the W-N and N-W setups, and that of LCP-GEM.

at lower $E_{\mathrm{d}}$ than that of LCP-GEM. We speculate that the discrepancy comes from the difference is shape of the equipotential plane around the GEM holes due to the different thickness of the electrodes. For thick electrode of RE-GEM, they works as a field shaper and significant part of the field lines in the drift region encounter the electrodes. Therefore some part of the signal electrons is caught by the thicker cathode of RE-GEM. As a result, a few signal electrons only pass through the RE-GEM holes. 


\section{Summary}

We succeeded in development of RE-GEM which has a fine pitch $(140 \mu \mathrm{m})$ and a thin insulator layer $(100 \mu \mathrm{m})$ between the resistive electrodes. The hole structure of RE-GEM is a single conical with the wider and narrower diameter of $80 \mu \mathrm{m}$ and $60 \mu \mathrm{m}$, respectively. The electrode thickness of RE-GEM was $25 \mu \mathrm{m}$. We succeeded to operate RE-GEM for the first time. The maximum gain of RE-GEM was about 600. The measurement of the dependence of gain on $E_{\mathrm{d}}$ shows that the electron transparency of RE-GEM is worse than that of LCP-GEM. We speculate the worse electron transparency of RE-GEM due to the thick electrodes.

\section{Acknowledgments}

This research and development of RE-GEM is supported by the Japan MPGD basic R\&D team. It is particularly notable, that Mr. Koshimuta's techniques at SciEnergy Co., Ltd. in Japan support our teams to produce RE-GEM.

\section{References}

[1] F. Sauli, GEM: A new concept for electron amplification in gas detectors, Nucl. Instrum. Meth. A 386 (1997) 531.

[2] V. Peskov and P. Fonte, Research on discharges in micropattern and small gap gaseous detectors, arXiv:0911.0463.

[3] A. Di Mauro et al., Development of innovative micropattern gaseous detectors with resistive electrodes and first results of their applications, Nucl. Instrum. Meth. A 581 (2007) 225 [arXiv:0706.0102].

[4] R. Oliveira, V. Peskov, F. Pietropaolo and P. Picchi, First Tests of Gaseous Detectors Made of a Resistive Mesh, arXiv:1002.1415.

[5] R. Akimoto, et al., Measurement of basic features of Thick-GEM and Resistive-GEM, 2010 JINST 5 P03002.

[6] T. Tamagawa et al., Development of gas electron multiplier foils with a laser etching technique, Nucl. Instrum. Meth. A 560 (2006) 418.

[7] T. Tamagawa et al., Development of thick-foil and fine-pitch GEMs with a laser etching technique, Nucl. Instrum. Meth. A 608 (2009) 390.

[8] S. Bachmann et al., Charge amplification and transfer processes in the gas electron multiplier, Nucl. Instrum. Meth. A 438 (1999) 376.

[9] F. Sauli, CERN Yellow Report, CERN 77-09, CERN, Geneva (1977).

[10] A. Bressan et al., High rate behavior and discharge limits in micropattern detectors, Nucl. Instrum. Meth. A 424 (1999) 321.

[11] A. Yoshikawa et al., Development of Gas Electron Multipliers with Resistive Kapton Electrodes, IEEE Conf. Rec. (2010) N66-2, 1873. 